\title{
Sex and Hemisphere - A Neglected, Nature-Determined Relationship in Acute Ischemic Stroke
}

\author{
Christian Hametner $^{\mathrm{a}}$ Peter Ringleb ${ }^{\mathrm{a}}$ Lars Kellert ${ }^{\mathrm{a}, \mathrm{b}}$ \\ a Department of Neurology, University of Heidelberg, Heidelberg, ${ }^{b}$ Department of Neurology, \\ Ludwig-Maximilians-University Munich, Munich, Germany
}

\section{Key Words}

Acute ischemic stroke - Intravenous thrombolysis . Coarsened exact matching $\cdot$ Sex $\cdot$ Hemisphere $\cdot$ Left hemispheric stroke $\cdot$ Right hemispheric stroke $\cdot$ Brain connectivity

\begin{abstract}
Background: Sex differences in the structural connectome of the brain are clinically highly relevant, but they have mostly been neglected in stroke trials. We investigated the impact of the interaction sex-by-hemisphere on outcome in stroke patients after intravenous thrombolysis (IVT). Methods: This is an observational study based on consecutively collected supratentorial stroke patients treated with IVT $(n=1,231)$. The 3-month modified Rankin scale (mRS) was estimated by adjusted binary (mRS 0-2 for good outcome) and ordinal regression analysis. As baseline characteristics differ substantially between the sexes, we aimed for better covariate balance by employing coarsened exact matching. Results: Sexby-hemisphere predicted good outcome in the entire cohort (726 left, 505 right hemispheric strokes, $p$ value interaction 0.032 ) and in the matched cohort (338 left, 273 right, $p$ val$u_{\text {interaction }}$ 0.003). Ordinal regression suggested a compara-

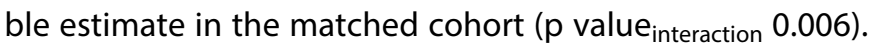
Further investigation revealed relevant between-sex and within-sex risk: right hemispheric strokes in men were 1.54 times (95\% confidence intervals (Cls) 1.15-2.01) more likely
\end{abstract}

than in women to achieve mRS 0-2. Women with right hemispheric strokes were 0.72 times $(95 \% \mathrm{Cl} 0.54-0.92)$ less likely to reach mRS 0-2 than women with left hemispheric strokes. Conversely, men with right hemispheric strokes were 1.35 times (95\% Cl 1.06-1.70) more likely to achieve mRS 0-2 than men with left hemispheric strokes. Conclusion: This study suggests that outcomes are different in both sexes after IVT when different hemispheres are affected. Further consideration of this hypothesis in clinical trials might help in guiding individualized, injury-specific treatment approaches for acute ischemic stroke.

(c) 2015 S. Karger AG, Basel

\section{Introduction}

Important evidence exists for sex-related brain dimorphism, for lateralization of brain functions and for sex differences in the structural connectome of the human brain $[1,2]$. Female brains are strongly connected between hemispheres, whereas intra-hemispheric communication is more prominent in male brains [2]. While most neuroscientific studies try to take these facts into consideration, both sex and hemisphere (right vs. left hemispheric stroke) have been commonly neglected in acute stroke trials. In fact, recommendations in current guidelines basically ignore these 2 factors and thus they are usually not taken into account in the decision-making process for acute stroke [3].

\section{KARGER 125}

(c) 2015 S. Karger AG, Base

$1015-9770 / 15 / 0402-0059 \$ 39.50 / 0$ 
The following facts should be considered with regard to the importance of hemisphere in stroke thrombolysis: the National Institutes of Health Stroke Scale (NIHSS) score has been reported to have an inherent substantial bias, making it more likely that patients with similar lesions in the left hemisphere versus the right hemisphere will be subjected to intervention [4-7]. Furthermore, medical attention and general stroke management have been shown to differ between hemispheres. Difficulties in recognizing stroke symptoms are more likely in right hemispheric lesions [8]. Moreover, there is uncertainty as to whether the hemisphere predicts outcome in patients treated with intravenous thrombolysis (IVT) and in placebo-treated patients $[5,7,9]$.

As for the importance of sex in stroke thrombolysis, post hoc analyses from clinical trials and from observational studies report heterogeneous results. Most studies found no differences in the 3-month outcome between women and men, but some report a disadvantage for women [10-14].

However, all these studies have one critical bias in common: since randomization for sex and hemisphere is (primarily) not possible, higher grade evidence is difficult to establish, leaving researchers to rely solely on a more robust analysis of available data. To address this issue, in addition to normal regression analysis, we performed a parallel analysis using coarsened exact matching (CEM) to find a better balance in the multidimensional distribution of covariates before analyzing the outcome parameters by an adjusted regression model. This 2-step approach is less prone to model misspecification and is even more robust than results based on the full unmatched data set [15-17].

We sought to illustrate how these 2 connectivity-relevant criteria, sex and hemisphere, modify outcome in acute ischemic stroke care after stroke thrombolysis.

\section{Methods}

\section{Database and Definitions}

From our local stroke database (prospectively collected and retrospectively analyzed data), we analyzed clinical and imaging data of all consecutive patients with supratentorial stroke who received IVT from 1998 to August $2013(\mathrm{n}=1,456)$. Our prospective local stroke database is managed and this study was performed according to the STROBE statement for reporting case-control studies [18]. Data were collected as part of the national and international quality control programs. The retrospective analysis of the data lacks any treatment influence and therefore written informed consent was waived. We excluded 30 instances of stroke mimic, 4 patients with missing follow-up imaging, 8 patients with missing 3-month follow-up, 28 patients with missing time-to-treatment information and 155 patients with at least one missing risk factor status from further analysis. The remaining 1,231 patients comprised the unmatched cohort. Outcome at 3 months was assessed using the modified Rankin scale (mRS). Good outcome was defined as mRS $0-2$. The definition of symptomatic intracerebral hemorrhage ( $\mathrm{sICH}$ ) according to the ECASS-II trial definition was applied [19].

\section{Coarsened Exact Matching}

The aim of matching is not to estimate, but rather to find a better balance in the multidimensional distribution of covariates. CEM [20] (or 'Cochran Exact Matching') is a matching method of the class monotonic imbalance bounding. This means that reducing an imbalance in the empirical distribution in one covariate has no effect on any other covariates chosen for balancing, which represents a clear advantage of CEM over other matching methods [21]. Briefly, the CEM algorithm consists of 3 steps. First, desired variables of all patients are coarsened temporarily. Second, all patients of the initial cohort are stratified on the basis of their coarsened variables. Third, only patients with strata containing at least one entry in both groups are kept; others are excluded. Additionally, a weighting variable is generated to equalize the number of entries in one stratum. This, in turn, reduces the degree of dependence on the estimation model of the outcome variable and therefore diminishes bias [17]. Of course, only observed variables can be accounted for in matching, and thus bias of omitted covariates cannot be eliminated. CEM was performed for the following variables: age, sex, $>1 / 3$ middle cerebral artery (MCA), hypertension, diabetes, atrial fibrillation, current smoking, congestive heart failure and hyperlipidemia. To measure the balancing performance, a multivariate imbalance measure L1 was introduced [20]. It is a relative magnitude depending on the individual data set and the chosen covariates. Ranging from 1 to 0 , a decrease (toward 0 ) in the L1 score yields a better balance.

\section{NIHSS Score and Hemisphere}

It is well known that the NIHSS implies a substantial bias because it favors left hemispheric stroke with respect to physicians' assessment of stroke severity [9] and with respect to lesion size [4, 5]. For example, a right hemispheric stroke that scores 8 points on the NIHSS is more likely to involve $>1 / 3$ of the territory of the MCA than a left hemispheric stroke with the same score, especially when the latter affects distinct areas of speech. Explicitly considering these matters, we bypassed the variable NIHSS (only) in the matching algorithm and introduced the variable infarct size $>1 / 3$ ' as a straightforward (less 'side-biased') measure to account for stroke severity, which one investigator (P.R.) estimated by qualitative means. This ensures a balanced distribution of strokes above and below this cut-off (1/3 of MCA territory) between the left and right side, which would less likely be the case when using NIHSS.

\section{Statistical Analysis and Variable Selection}

Groups of baseline characteristics were compared with the Student's t test, the Mann-Whitney U test or the Fisher's exact test, as appropriate, and matched weights were accounted for in matched group comparisons. The outcome distribution on the $\mathrm{mRS}$ is given for both the entire and the matched cohort with subgroups of sexspecific hemispheres, respectively.
60

Cerebrovasc Dis 2015;40:59-66

DOI: $10.1159 / 000430999$
Hametner/Ringleb/Kellert 
Table 1. Baseline characteristics for hemisphere in entire and matched cohort

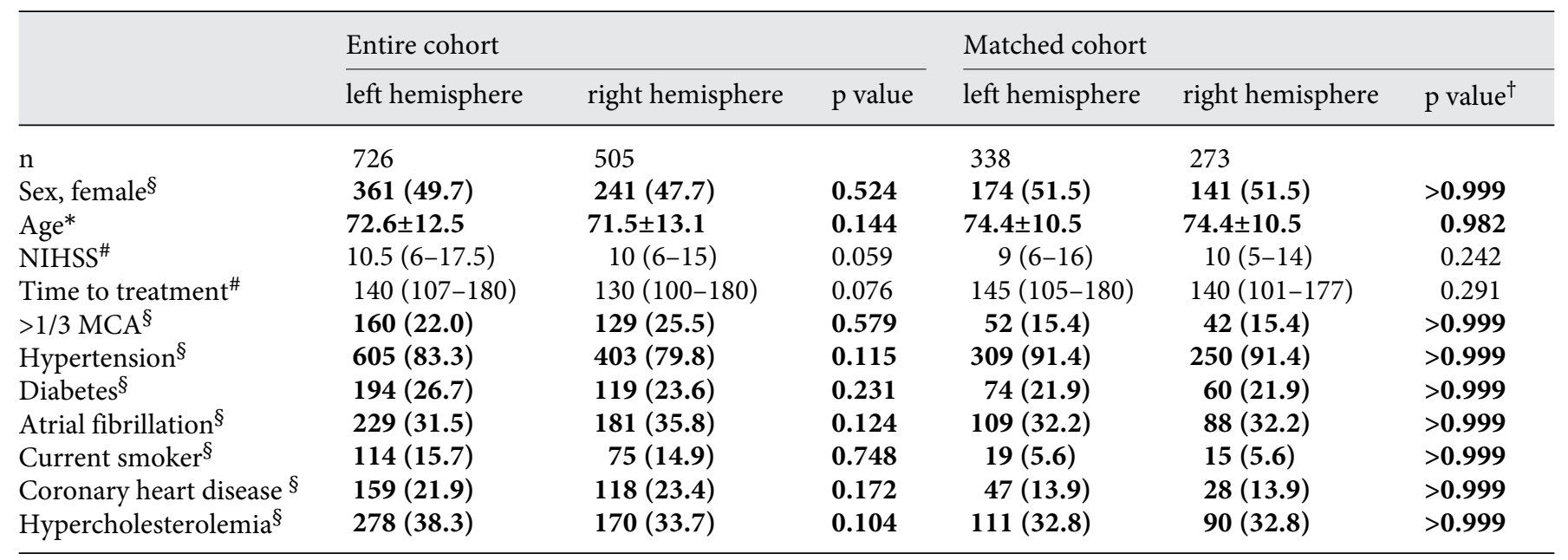

Numbers are mean \pm SD or median $(25 / 75$ IQR) or counts (percentages). Matched variables are marked bold. * Student's t test; \# Mann-Whitney U test; ${ }^{\S}$ Chi-square/Fisher exact test; ${ }^{\dagger}$ considering matched weights. NIHSS = National Institutes of Health Stroke Scale; MCA = middle cerebral artery.

To investigate the relationship between sex and hemisphere, interaction terms were integrated into the binomial logistic regression models by following the recommendations of Hosmer et al. [22]. The main effects model consisted of the following variables, which are also listed in table 1 , namely, $\operatorname{sex}($ female $=1$, male $=0$ ), age (as continuous variable), hemisphere (left $=1$, right $=0$ ), infarct size $>1 / 3(>1 / 3$ of MCA territory $=1)$, NIHSS (as continuous variable), time to treatment $(<271 \mathrm{~min}=1)$, atrial fibrillation (disease status yes $=1)$, coronary heart disease (disease status yes $=1$ ), hypertension (disease status yes $=1$ ), diabetes (disease status yes = 1 ), hypercholesterolemia (disease status yes $=1$ ), current smoking (disease status yes $=1$ ) and sICH (disease status yes $=1$ ). Variable selection was based not only on model fit statistics, but also on pathophysiological considerations regarding stroke in general with an emphasis on sex-specific factors. Importantly, all pathophysiological factors included are well known to differ between the sexes and therefore need to be considered in the model. All possible 2-way interactions between these variables were then entered separately in the main effects model. Meaningful interactions that contributed at a significance level of $p<0.1$ could be added to the preliminary final model. This ensured that interactions that would only emerge in the presence of others were accounted for. In the last step of forming the final model, we only added interactions that contributed at a significance level of $p<0.01$. Aside from interactions, a level of $\mathrm{p}<0.05$ was considered significant.

For a better understanding of the interaction term sex-byhemisphere, a 4-factor variable of sex and hemisphere was created (female-right, female-left, male-right, male-left; reference category: male-right). This variable entered the final regression model instead of the variables sex, hemisphere and the interaction sex-byhemisphere. The final model is reported using odds ratios (ORs) and $95 \%$ confidence intervals (CIs). For reasons of transparency, we also report the adjustment evolution for the binary model (good outcome) starting with the univariate model as the supplementary material.

Sex and Hemisphere in Stroke

Thrombolysis
Using the final model of mRS 0-2 (fig. 2), simulations studies were performed following the approach previously described by King et al. [23]. To increase reliability, the simulations were based on $n=10,000$ draws instead of the standard $(n=1,000)$. Furthermore, contrasts of hemispheres within sex and between sex were calculated by dividing the adjusted means (adjusted for the same factors as in the final model, namely, age, infarct size $>1 / 3$, NIHSS, time to treatment, atrial fibrillation, coronary heart disease, hypertension, diabetes, hypercholesterolemia, current smoking and sICH) and reporting as adjusted risk ratios (RRs) with 95\% CI. Statistical analysis was performed using R [24-26] and SPSS (SPSS Inc., 21.0 for Windows).

\section{Results}

\section{Baseline Characteristics and Outcome Distribution}

The covariate balance in the baseline characteristics substantially improved from the entire cohort (726 left and 505 right hemispheric strokes) to the matched cohort (338 left and 273 right hemispheric strokes; L1 improvement from 0.540 to 0.131 ; table 1 ).

The outcome distribution is given according to $\mathrm{mRS}$ for the entire (fig. 1a) and the matched cohort (fig. 1b) with relevant subgroups of the interaction sex-by-hemisphere.

\section{Regression Analysis}

In the entire cohort, regression analysis for good outcome showed NIHSS (OR 0.84, 95\% CI 0.82-0.87), infarct size $>1 / 3$ MCA (OR 0.15, 95\% CI 0.09-0.23), age 
Fig. 1. Three-month mRS distribution of the entire (a) and the matched cohort (b) of the 4 sex-by-hemisphere subgroups, respectively.

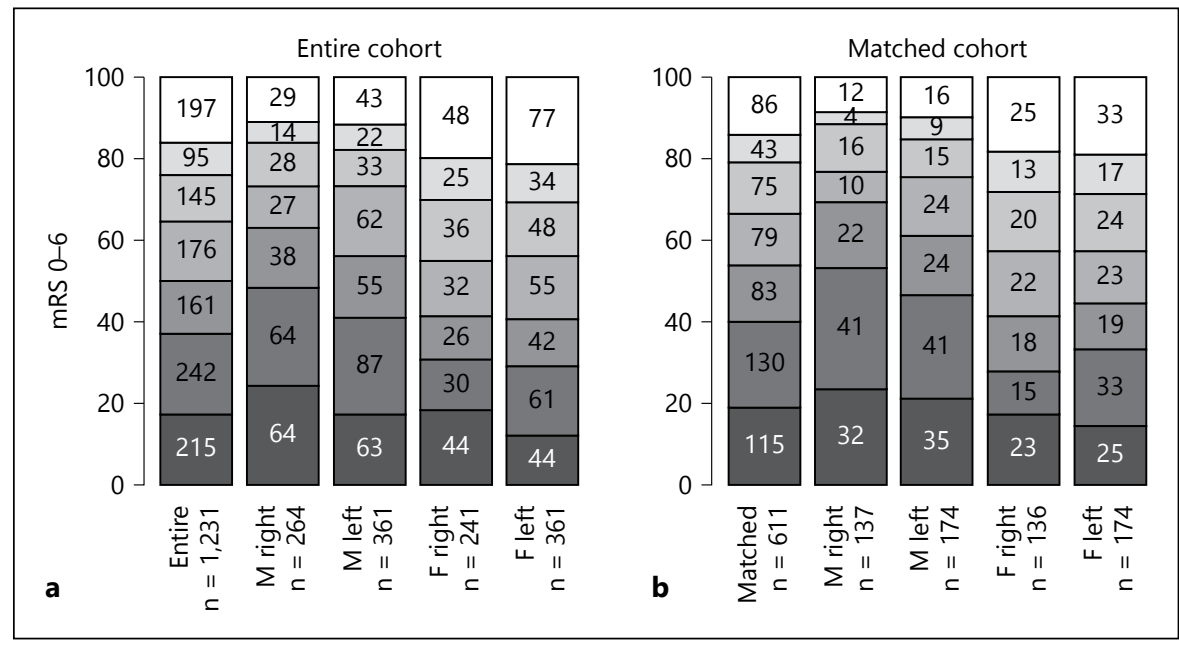

Table 2. Binomial and ordinal adjusted * logistic regression models in entire and matched cohort for outcomes of mRS $0-2$ and outcomes according to the ordinal scale of the mRS

\begin{tabular}{|c|c|c|c|c|c|c|c|c|}
\hline & \multicolumn{4}{|l|}{ Entire cohort } & \multicolumn{4}{|l|}{ Matched cohort } \\
\hline & \multicolumn{2}{|l|}{ mRS 0-2 } & \multicolumn{2}{|l|}{ ordinal $^{\#}$} & \multicolumn{2}{|l|}{ mRS 0-2 } & \multicolumn{2}{|l|}{ ordinal $^{\#}$} \\
\hline & OR $(95 \% \mathrm{CI})$ & $\mathrm{p}_{\text {interaction }}$ & OR (95\% CI) & $\mathrm{p}_{\text {interaction }}$ & OR (95\% CI) & $\mathrm{p}_{\text {interaction }}$ & OR (95\% CI) & $\mathrm{p}_{\text {interaction }}$ \\
\hline Sex-by-hemisphere & & 0.032 & & 0.168 & & 0.003 & & 0.006 \\
\hline Male right (reference) & - & & - & & - & & - & \\
\hline Male left & $0.60(0.39-0.91)$ & & $0.73(0.55-0.98)$ & & $0.54(0.29-0.97)$ & & $0.64(0.42-0.98)$ & \\
\hline Female right & $0.51(0.31-0.81)$ & & $0.71(0.51-0.99)$ & & $0.43(0.22-0.83)$ & & $0.60(0.38-0.96)$ & \\
\hline Female left & $0.59(0.38-0.90)$ & & $0.70(0.52-0.95)$ & & $0.85(0.45-1.60)$ & & $0.88(0.56-1.37)$ & \\
\hline NIHSS & $0.84(0.82-0.87)$ & & $0.87(0.85-0.89)$ & & $0.83(0.80-0.87)$ & & $0.86(0.84-0.89)$ & \\
\hline$>1 / 3 \mathrm{MCA}$ & $0.15(0.09-0.23)$ & & $0.20(0.15-0.27)$ & & $0.14(0.05-0.31)$ & & $0.23(0.14-0.37)$ & \\
\hline Age & $0.94(0.93-0.95)$ & & $0.96(0.95-0.96)$ & & $0.93(0.90-0.95)$ & & $0.95(0.93-0.96)$ & \\
\hline Time to treatment & $1.04(0.63-1.72)$ & & $0.83(0.59-1.17)$ & & $1.13(0.53-2.43)$ & & $0.92(0.54-1.55)$ & \\
\hline sICH & $0.02(0.00-0.11)$ & & $0.07(0.03-0.13)$ & & $0.03(0.00-0.18)$ & & $0.04(0.01-0.11)$ & \\
\hline Current smoking & $0.51(0.33-0.78)$ & & $0.71(0.53-0.96)$ & & $0.34(0.11-1.08)$ & & $0.36(0.17-0.76)$ & \\
\hline Atrial fibrillation & $0.90(0.65-1.25)$ & & $0.84(0.67-1.06)$ & & $0.85(0.53-1.38)$ & & $0.64(0.45-0.92)$ & \\
\hline Hypertension & $0.74(0.49-1.13)$ & & $0.84(0.63-1.12)$ & & $0.50(0.15-1.47)$ & & $0.78(0.42-1.46)$ & \\
\hline Hypercholesterolemia & $1.19(0.87-1.65)$ & & $1.15(0.92-1.45)$ & & $0.96(0.60-1.54)$ & & $0.97(0.69-1.38)$ & \\
\hline Coronary heart disease & $1.09(0.76-1.58)$ & & $0.93(0.72-1.21)$ & & $1.67(0.87-3.24)$ & & $0.90(0.55-1.45)$ & \\
\hline Diabetes & $0.72(0.51-1.01)$ & & $0.78(0.61-0.99)$ & & $0.89(0.53-1.48)$ & & $1.02(0.70-1.47)$ & \\
\hline
\end{tabular}

Significant estimates $(\mathrm{p}<0.05)$ are marked bold. * Adjusted for sex, age, NIHSS, $>1 / 3 \mathrm{MCA}$, hypertension, diabetes, atrial fibrillation, time to treatment, coronary heart disease, hypercholesterolemia, $\mathrm{sICH} .{ }^{\#}$ mRS 0 as reference category. $>1 / 3 \mathrm{MCA}=$ Greater than one third of middle cerebral artery territory; $\mathrm{sICH}=$ symptomatic intracerebral hemorrhage (ECASS II definition); $\mathrm{OR}=$ odds ratio; $\mathrm{CI}=$ confidence interval.

(OR 0.94, 95\% CI 0.93-0.95), sICH (OR 0.02, 95\% CI $0.00-0.11$ ) and current smoking (OR 0.51, 95\% CI 0.330.78 ) as negative predictors. Ordinal regression suggested similar independent predictors (table 2).

The results for the matched cohort for good outcome and the binomial and ordinal regression model were comparable regarding generally accepted outcome predictors.

\section{Regression Analysis - Sex and Hemisphere}

The interaction of sex-by-hemisphere contributed significantly to the final regression model of good outcome in the entire cohort (OR 1.94, 95\% CI 1.06-3.56) and in the matched cohort (OR 3.64, 95\% CI 1.58-8.54; table 2). For easier interpretation, a 4 -factor variable (female-left, female-right, male-left and male-right as reference category) was introduced. Significant predictors for good 
Fig. 2. Interaction plot of sex-by-hemisphere in the entire (left part of the figure) and the matched cohort (right part), demonstrating the predicted probability of reaching good outcome. The lower part of the figure describes the corresponding adjusted risk ratios.

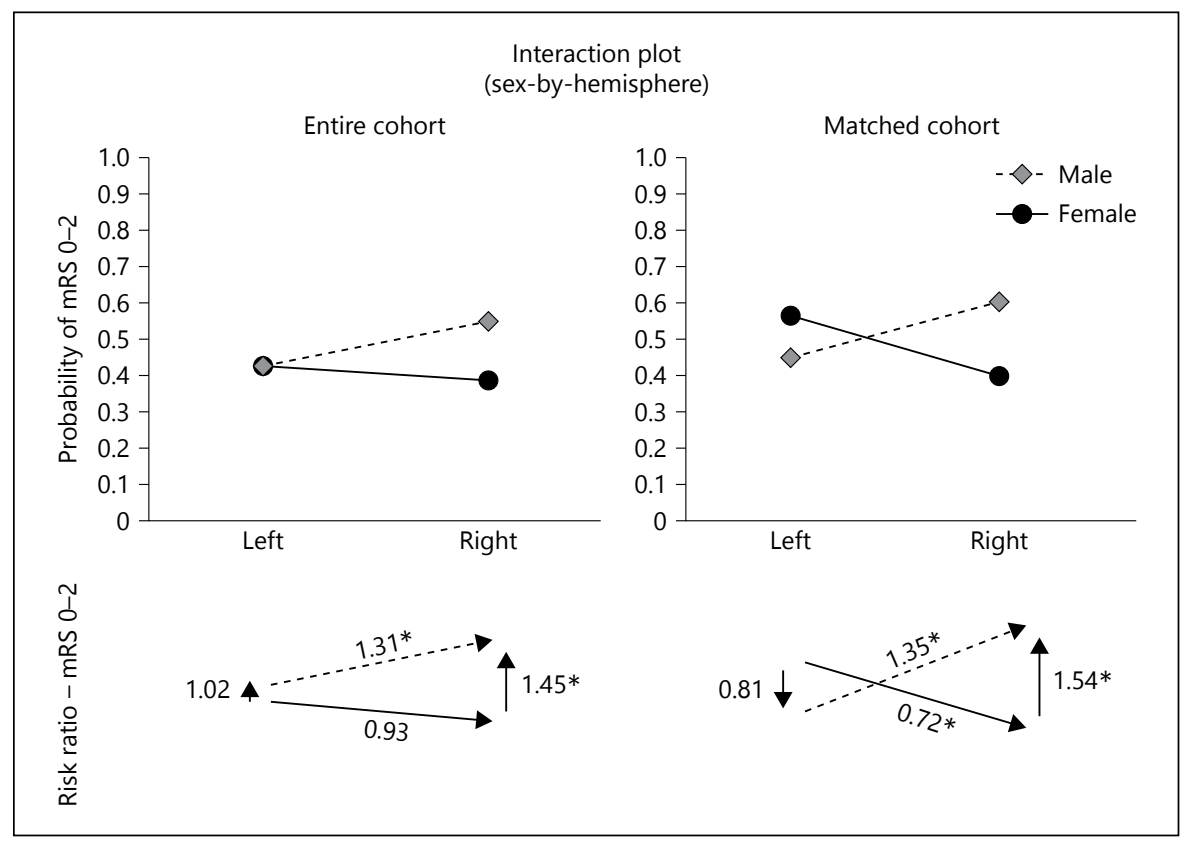

outcome were observed in the entire cohort for femaleleft (OR 0.59, 95\% CI 0.38-0.90), female-right (OR 0.51, 95\% CI 0.31-0.81) and male-left (OR 0.60, 95\% CI 0.390.91 ). In the matched cohort, female-right (OR $0.43,95 \%$ CI 0.22-0.83) and male-left (OR 0.54, 95\% CI 0.29-0.97) estimates were comparable, whereas female-left (OR 0.85 , 95\% CI 0.45-1.60) scored differently (reference category: men-right). A detailed logistic regression including stepwise evolution of the model is presented in the online supplement (online suppl. tables 1 and 2; for all online suppl. material, see www.karger.com/doi/10.1159/000 430999).

Ordinal regression confirmed the influence of the interaction of sex-by-hemisphere over a wide $\mathrm{mRS}$ range (OR 1.34, 95\% CI 0.88-2.04), which reached significance (OR 2.27, 95\% CI 1.27-4.10) in the matched cohort. The 4-factor variable (female-left, female-right, male-left, male-right (reference category)) yielded results comparable to those in the binominal regression model (table 2).

\section{Between-Sex Contrast}

A between-sex difference was obvious for the right hemisphere but not for the left hemisphere (fig. 2): in men, outcome after right hemispheric strokes was 1.54 times more likely to be good than in women (entire cohort RR 1.45 (95\% CI 1.16-1.78), matched cohort RR 1.54 (95\% CI 1.15-2.01)). The likelihood for left hemispheric stroke in women was not significantly different from the

Sex and Hemisphere in Stroke

Thrombolysis risk in men of reaching a good outcome 90 days after stroke (entire cohort RR 1.02 (95\% CI 0.84-1.22), matched cohort RR 0.81 (95\% CI 0.62-1.03)).

\section{Within-Sex Contrast}

A within-sex difference of hemisphere demonstrated a reversed effect in women and men (fig. 2): women who were affected by stroke in the right hemisphere were 0.72 times less likely of reaching good outcome than were women in whom left hemisphere was affected. The estimate for the entire cohort pointed toward this (RR 0.92 (95\% CI 0.74-1.14)) and reached significance in the matched cohort (RR 0.72 (95\% CI 0.54-0.92)). Conversely with women, men who were affected by stroke in the right hemisphere were 1.35 times more likely of achieving good outcome than were men in whom the left hemisphere was affected (entire cohort RR 1.31 (95\% CI 1.081.56), matched cohort RR 1.35 (95\% CI 1.06-1.70)).

\section{Interaction by Range of NIHSS Distribution}

To demonstrate the impact of stroke severity on the between-sex and within-sex hemisphere effect, the predicted probability of reaching good outcome by range of NIHSS is presented for the matched cohort (fig. 3).

\section{Influence of NIHSS}

To investigate a possible influence of NIHSS-inherent hemisphere bias (favoring left hemispheric stroke [4-7]) on this interaction effect, the interaction sex-by-hemi- 


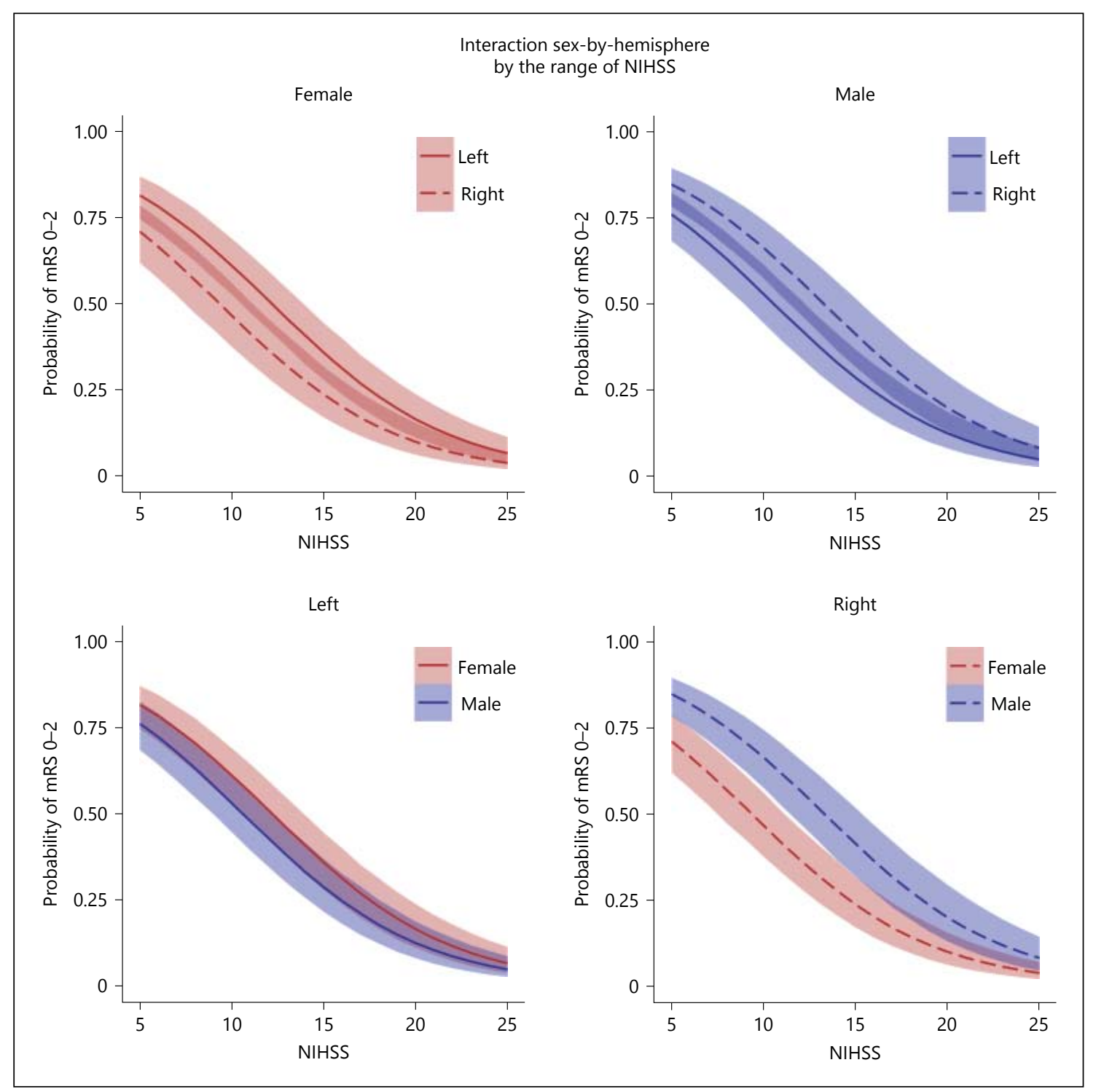

Fig. 3. Illustration of predicted probability of reaching mRS $0-2$ by range of NIHSS (demonstrated is sector 5-25) describes the relationship between sex and hemisphere in the matched cohort within the sexes (left vs. right, upper part of the figure) and between the sexes (female vs. male, lower part).

sphere of the adjusted final binary outcome model as calculated above was compared to a model without NIHSS. The analysis indicated that the interaction was not evoked by the hemisphere bias inherent within the NIHSS: (1) the estimate of reaching mRS $0-2$ of the interaction sex-by-hemisphere and (2) the direction of this interaction in the model omitting NIHSS demonstrated comparable results to the full model in both the analysis of the entire and the matched cohort (online suppl. tables 3 and 4).

\section{Discussion}

In the present study, we show a strong association between the interaction of sex-by-hemisphere and outcome in patients treated with IVT. Upon further investigation, between-sex and within-sex variation revealed relevant effects on outcome - men with right hemispheric lesions fared best in this cohort, while outcome in women with right hemispheric stroke was worst.

Put into the context of previous studies, which focused either on sex [10-14] or hemisphere [7, 9] separately, this 
study - for the first time - accounts for the relationship between the 2 factors and may partially explain the heterogeneous results concerning the impact of sex in stroke thrombolysis. As in other neuroscientific studies, the relationship between sex and hemisphere might play a more profound role in stroke thrombolysis than expected thus far.

Again, this analysis found men with right hemispheric strokes to be 1.54 times more likely than women with right hemispheric strokes to reach a good outcome (fig. 1). Aside from this between-sex performance, a relevant within-sex difference of hemisphere was found in both sexes. This emphasizes that not only the individual term, but especially the relationship between sex and hemisphere is important to consider when determining stroke outcome. According to ordinal regression in the matched cohort, the effect of sex-by-hemisphere appeared to be present over the full $\mathrm{mRS}$ range (table 2). Investigating the relationship by range of the NIHSS revealed that differences for probability of reaching good outcome peaked at the NIHSS level representing moderate to severe strokes (fig. 3). This finding is of great relevance, because this level of stroke severity targets the potential population of future clinical stroke trials. Accounting for sex-by-hemisphere in future trials could easily be accomplished preferably by performing block randomization of the 4 groups of this interaction.

Overall, deductions cannot be made about underlying pathophysiological mechanisms using this study design. Recent studies using diffusion-tensor imaging [27] investigated the structural connectome of the human brain and proposed that female brains connect more strongly between hemispheres, whereas intrahemispheric communication is more prominent in male brains $[2,28]$. This theory is in line with long-standing concepts of hemispheric specialization of language function $[28,29]$. In addition to these findings in resting state, task-dependent recruitment of circuits (functional connectome) may also differ. One study investigated interhemispheric interactions during phonological processing and suggested that the potential for interhemispheric cooperation is higher in females [30]. Another study investigating figurative language comprehension found greater overall activation in females [31].

It is not known how and if this 'wider' circuit integration of language in females is of benefit when diaschisis occurs during recovery after network disruption as a result of stroke [32]. Outcome was assessed in this observational study at 90 days after stroke onset. In this time period, very different mechanisms such as post-stroke depression [33] or post-stroke memory decline [34] in the

Sex and Hemisphere in Stroke

Thrombolysis brain hemispheres in women and men may also be responsible for the fact that recovery in women with right hemispheric strokes was worst in our cohort. Further investigation is needed to confirm the consistency of this finding in long-term outcomes. Additional clinical trials that consider the present hypothesis might help in guiding individualized, injury-specific treatment approaches [35] for acute ischemic stroke.

Our study has at least the following limitations: data although prospectively collected - were derived from a single stroke center in a period of about 15 years and were retrospectively analyzed. Therefore, with our study design, we cannot conclude that this finding causally influences outcome. External validation of this hypothesis is needed. The matching process aimed to diminish statistical bias [17]. However, selecting matching parameters may in itself introduce bias as a compromise is always made between finding the optimal match and a meaningful size of the matched cohort.

\section{Conclusion}

This study suggests that the 2 sexes achieve different outcomes following IVT when different hemispheres are affected. It emphasizes that not only the individual terms, but also factors of the relationship sex-by-hemisphere are important to consider when determining stroke outcome. This is clinically highly relevant and future studies should consider this possible effect modifier.

\section{Author Contributions}

L.K. and C.H. developed the idea for this study by interaction of intellectual content, collected and analyzed clinical data, performed the statistical analysis and contributed equally to writing the manuscript. P.R. collected and analyzed clinical data, supervised data acquisition and edited the manuscript for intellectual content.

\section{Disclosure Statement}

L.K. and C.H. declare no conflicts of interest.

P.R. reports personal fees from travel support and speaker honoraria from Boehringer Ingelheim, outside the submitted work; he is national coordinator of the SITS registry and member of the steering committee of the ECASS- 4 trial.

\section{Sources of Funding}

None. 


\section{References}

1 Cahill L: Why sex matters for neuroscience. Nat Rev Neurosci 2006;7:477-484.

2 Ingalhalikar M, Smith A, Parker D, Satterthwaite TD, Elliott MA, Ruparel K, Hakonarson H, Gur RE, Gur RC, Verma R: Sex differences in the structural connectome of the human brain. Proc Natl Acad Sci U S A 2014;111: 823-828.

3 Adams HP Jr, del Zoppo G, Alberts MJ, Bhatt DL, Brass L, Furlan A, Grubb RL, Higashida RT, Jauch EC, Kidwell C, Lyden PD, Morgenstern LB, Qureshi AI, Rosenwasser RH, Scott PA, Wijdicks EF; American Heart Association; American Stroke Association Stroke Council; Clinical Cardiology Council; Cardiovascular Radiology and Intervention Council; Atherosclerotic Peripheral Vascular Disease and Quality of Care Outcomes in Research Interdisciplinary Working Groups: Guidelines for the early management of adults with ischemic stroke: a guideline from the American heart association/American stroke association stroke council, clinical cardiology council, cardiovascular radiology and intervention council, and the atherosclerotic peripheral vascular disease and quality of care outcomes in research interdisciplinary working groups: the American academy of neurology affirms the value of this guideline as an educational tool for neurologists. Stroke 2007;38:1655-1711.

4 Woo D, Broderick JP, Kothari RU, Lu M, Brott T, Lyden PD, Marler JR, Grotta JC: Does the national institutes of health stroke scale favor left hemisphere strokes? NINDS t-PA Stroke Study Group. Stroke 1999;30:23552359.

5 Fink JN, Selim MH, Kumar S, Silver B, Linfante I, Caplan LR, Schlaug G: Is the association of national institutes of health stroke scale scores and acute magnetic resonance imaging stroke volume equal for patients with right- and left-hemisphere ischemic stroke? Stroke 2002;33:954-958.

6 Lee M, Saver JL, Alger JR, Hao Q, Salamon N, Starkman S, Ali LK, Ovbiagele B, Kim D, Villablanca JP, Froehler MT, Tenser MS, Liebeskind DS: Association of laterality and size of perfusion lesions on neurological deficit in acute supratentorial stroke. Int J Stroke 2012; 7:293-297.

7 Blondin NA, Staff I, Lee N, McCullough LD: Thrombolysis in right versus left hemispheric stroke. J Stroke Cerebrovasc Dis 2010;19:269_ 272.

8 Foerch C, Misselwitz B, Sitzer M, Berger K, Steinmetz H, Neumann-Haefelin T; Arbeitsgruppe Schlaganfall Hessen: Difference in recognition of right and left hemispheric stroke. Lancet 2005;366:392-393.

9 Di Legge S, Saposnik G, Nilanont Y, Hachinski V: Neglecting the difference: does right or left matter in stroke outcome after thrombolysis? Stroke 2006;37:2066-2069.
10 Kent DM, Price LL, Ringleb P, Hill MD, Selker HP: Sex-based differences in response to recombinant tissue plasminogen activator in acute ischemic stroke: a pooled analysis of randomized clinical trials. Stroke 2005;36:6265.

11 Kent DM, Buchan AM, Hill MD: The gender effect in stroke thrombolysis: of CASES, controls, and treatment-effect modification. Neurology 2008;71:1080-1083.

12 Lorenzano S, Ahmed N, Falcou A, Mikulik R, Tatlisumak T, Roffe C, Wahlgren N, Toni D; SITS Investigators: Does sex influence the response to intravenous thrombolysis in ischemic stroke?: answers from safe implementation of treatments in stroke-international stroke thrombolysis register. Stroke 2013;44: 3401-3406.

13 Shobha N, Sylaja PN, Kapral MK, Fang J, Hill $\mathrm{MD}$; Investigators of the Registry of the Canadian Stroke Network: Differences in stroke outcome based on sex. Neurology 2010;74:767-771.

14 Elkind MS, Prabhakaran S, Pittman J, Koroshetz W, Jacoby M, Johnston KC; GAIN Americas Investigators: Sex as a predictor of outcomes in patients treated with thrombolysis for acute stroke. Neurology 2007;68:842848.

15 Rubin DB: The use of matched sampling and regression adjustment to remove bias in observational studies. Biometrics 1973;29:185203.

16 Rubin DB, Thomas N: Combining propensity score matching with additional adjustments for prognostic covariates. J Am Stat Assoc 2000;95:573-585.

17 Ho D, Imai K, King G, Stuart EA: Matching as nonparametric preprocessing for reducing model dependence in parametric causal inference. Polit Anal 2007;15:199-236.

18 von Elm E, Altman DG, Egger M, Pocock SJ, Gøtzsche PC, Vandenbroucke JP: The strengthening the reporting of observational studies in epidemiology (STROBE) statement: guidelines for reporting observational studies. Revista Espanola de Salud Publica 2008;82:251-259.

19 Hacke W, Kaste M, Fieschi C, von Kummer R, Davalos A, Meier D, Larrue V, Bluhmki E, Davis S, Donnan G, Schneider D, Diez-Tejedor E, Trouillas P: Randomised double-blind placebo-controlled trial of thrombolytic therapy with intravenous alteplase in acute ischaemic stroke (ECASS II). Second EuropeanAustralasian acute stroke study investigators. Lancet 1998;352:1245-1251.

20 Iacus SM, King G, Porro G: Causal inference without balance checking: coarsened exact matching. Polit Anal (Winter 2012);20:1-24. DOI:10.1093/pan/mpr013 (first published online August 23, 2011.
21 King G, Nielsen R, Coberley C, Pope JE, Wells A: Comparative Effectiveness of Matching Methods for Causal Inference, 2011.

22 Hosmer DW Jr, Lemeshow S, Sturdivant RX: Applied Logistic Regression, ed 3. John Wiley \& Sons, 2013

23 King G, Tomz M, Wittenberg J: Making the most of statistical analyses: improving interpretation and presentation. Am J Polit Sci 2000;44:341-355.

24 R Development Core Team: R: A Language and Environment for Statistical Computing. Vienna, Austria, R Foundation for Statistical Computing, 2013.

25 Imai K, King G, Lau O: Logit: logistic regression for dichotomous dependent variables; in Imai K, King G, Lau O (eds): Zelig: Everyone's Statistical Software. http://gking.harvard. edu/zelig, 2013.

26 King G, Imai K, King G, Stuart EA: MatchIt: Nonparametric Preprocessing for Parametric Causal Inference, 2007.

27 Basser PJ, Mattiello J, LeBihan D: MR diffusion tensor spectroscopy and imaging. Biophys J 1994;66:259-267.

28 Jahanshad N, Aganj I, Lenglet C, Joshi A, Yan J, Barysheva M, McMahon KL, de Zubicaray GI, Martin NG, Wright MJ, Toga AW, Sapiro G, Thompson PM: Sex differences in the human connectome: 4-tesla high angular resolution diffusion imaging (HARDI) tractography in 234 young adult twins. IEEE International Symposium, 2011, pp 939-943.

29 Shaywitz BA, Shaywitz SE, Pugh KR, Constable RT, Skudlarski P, Fulbright RK, Bronen RA, Fletcher JM, Shankweiler DP, Katz L, et al: Sex differences in the functional organization of the brain for language. Nature 1995; 373:607-609.

30 Bitan T, Lifshitz A, Breznitz Z, Booth JR: Bidirectional connectivity between hemispheres occurs at multiple levels in language processing but depends on sex. J Neurosci 2010;30: 11576-11585.

31 Kana RK, Murdaugh DL, Wolfe KR, Kumar SL: Brain responses mediating idiom comprehension: gender and hemispheric differences. Brain Res 2012;1467:18-26.

32 Carrera E, Tononi G: Diaschisis: past, present, future. Brain 2014;137:2408-2422.

33 Mazure CM, Weinberger AH, Pittman B, Sibon I, Swendsen J: Gender and stress in predicting depressive symptoms following stroke. Cerebrovasc Dis 2014;38:240-246.

34 Wang Q, Mejía-Guevara I, Rist PM, Walter S, Capistrant BD, Glymour MM: Changes in memory before and after stroke differ by age and sex, but not by race. Cerebrovasc Dis 2014;37:235-243.

35 Silasi G, Murphy TH: Stroke and the connectome: how connectivity guides therapeutic intervention. Neuron 2014;83:1354-1368. 